This item was submitted to Loughborough's Research Repository by the author.

Items in Figshare are protected by copyright, with all rights reserved, unless otherwise indicated.

\title{
Structural and chemical characterization of the back contact region in high efficiency CdTe solar cells
}

PLEASE CITE THE PUBLISHED VERSION

http://dx.doi.org/10.1109/PVSC.2015.7355622

PUBLISHER

(C) IEEE

VERSION

AM (Accepted Manuscript)

LICENCE

CC BY-NC-ND 4.0

\section{REPOSITORY RECORD}

Abbas, Ali, Daniel M. Meysing, Jiaojiao Li, Joseph D. Beach, Teresa M. Barnes, and Colin A. Wolden. 2019. "Structural and Chemical Characterization of the Back Contact Region in High Efficiency Cdte Solar Cells". figshare. https://hdl.handle.net/2134/20467. 


\title{
Structural and Chemical Characterization of the Back Contact Region in High Efficiency CdTe Solar Cells
}

\author{
A. Abbas, ${ }^{1}$ D. M. Meysing, ${ }^{2,3}$ J. Li, ${ }^{2}$ J.D. Beach, ${ }^{2}$ T. M. Barnes, ${ }^{3}$ J.M. Walls, ${ }^{1}$ and C. A. Wolden ${ }^{2}$ \\ ${ }^{1}$ Loughborough University, Loughborough, Leicestershire LE11 3TU, United Kingdom; \\ ${ }^{2}$ Colorado School of Mines, Golden, Colorado, 80401, USA; \\ ${ }^{3}$ National Renewable Energy Laboratory, Golden, Colorado, 80401, USA
}

\begin{abstract}
Cadmium telluride (CdTe) is the leading commercialized thin-film photovoltaic technology. Copper is commonly used in back contacts to obtain high efficiency, but has also been implicated as a harmful factor for device stability. Thus it is critical to understand its composition and distribution within complete devices. In this work the composition and structure of the back contact region was examined in high efficiency devices ( 16\%) contacted using a ZnTe:Cu buffer layer followed by gold metallization. The microstructure was examined in the asdeposited state and after rapid thermal processing (RTP) using high resolution transmission electron microscopy and EDX chemical mapping. After RTP the ZnTe exhibits a bilayer structure with polycrystalline, twinned grains adjacent to $\mathrm{Au}$ and an amorphous region adjacent to $\mathrm{CdTe}$ characterized by extensive Cd-Zn interdiffusion. The copper that is co-deposited uniformly within ZnTe is found to segregate dramatically after RTP activation, either collecting near the $\mathrm{ZnTe} / \mathrm{Au}$ interface or forming CuxTe clusters in CdTe at defects or grain boundaries near the interface with ZnTe. Chlorine, present throughout CdTe and concentrated at grain boundaries, does not penetrate significantly into the back contact region during RTP activation.
\end{abstract}

Index Terms - CdTe solar cells, TEM, back contact, copper

\section{INTRODUCTION}

With an ideal band gap $(\sim 1.45 \mathrm{eV})$ and a large absorption coefficient $\left(>10^{4} \mathrm{~cm}^{-1}\right)$ CdTe has emerged as the leading thin film photovoltaic (PV) technology with record efficiency currently at $21.5 \%$ [1]. Most recent advances have been primarily due to improvements in short circuit current density $\left(\mathrm{J}_{\mathrm{sc}}\right)$ and fill factor $(\mathrm{FF})$, whereas the open circuit voltage $\left(\mathrm{V}_{\mathrm{oc}}\right)$ values have remained largely unchanged [2]. Good ohmic contact with CdTe is a requirement for high $\mathrm{V}_{\text {oc }}$, which is challenging due to its low electron affinity. A common strategy to address this problem is through the insertion of a thin layer between the CdTe and metal contact [3]. A commonly used back buffer layer is copper doped zinc telluride (ZnTe:Cu) [4]. First Solar Inc. recently credited the use of ZnTe buffer layers for recent improvements in module reliability and stability [5].

Copper degenerately dopes this layer, which narrows the barrier width and permits electron tunneling, creating a quasiohmic contact [6]. However $\mathrm{Cu}$ has also been correlated with deep level defect, reduced carrier lifetime, and stability issues [7]. With copper having both positive and deleterious effects it is critical to precisely control both its amount and spatial distribution in order to obtain high efficiency. We have recently introduced a back contact procedure in which $\mathrm{ZnTe}: \mathrm{Cu}$ is coevaporated at low temperature followed by activation using

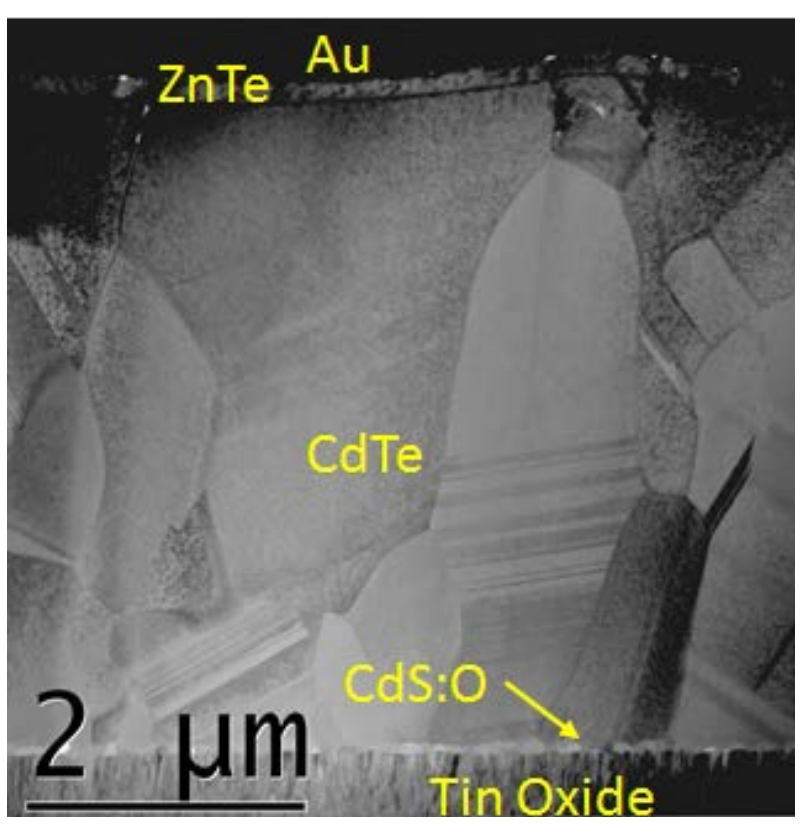

Fig. 1. Bright field TEM image of the sample showing the complete device.

rapid thermal processing (RTP) [8, 9]. In this work, high resolution transmission electron microscopy (HR-TEM) coupled with elemental analysis is applied to understand the microstructure and composition of the back contact region in optimally processed devices.

\section{EXPERIMENTAL}

\section{A. Device Fabrication}

CdTe solar cells were fabricated using the superstrate architecture. Chemical vapor deposited fluorine-doped tin oxide (FTO), intrinsic tin oxide (TO), reactively sputtered CdS:O, close-spaced sublimated CdTe, and an evaporated $\mathrm{ZnTe}: \mathrm{Cu} /$ gold back-contact were deposited sequentially on Corning 7059 glass. Deposition of the FTO/TO/CdS:O/CdTe layers occurred at the National Renewable Energy Lab (NREL) [10]. Application of the back contact and RTP activation was conducted at the Colorado School of Mines (CSM) [8]. In this work we compare the composition and structure of the back 
contact region in nominally identical devices, one in the asdeposited state and a second that was provided with an optimal RTP treatment. Figure 1 displays a bright field TEM cross section image of the latter, which displayed power conversion efficiencies of $\sim 16 \%$.

\section{B. Characterization}

Transmission electron microscopy was the tool used to investigate the detailed microstructure of the cells using the facilities at Loughborough. TEM samples were prepared by focused ion beam (FIB) milling using a dual beam FEI Nova 600 nanolab. A standard in situ lift out method described previously [11] was employed for sample preparation. High resolution TEM imaging was carried out in a FEI Technai F20 equipped with an Oxford Instruments X-Max 80 silicon drift detector (SDD) energy dispersive $\mathrm{X}$-ray detector (EDX). EDX was used to produce chemical distribution maps of the cells as well as line scans and point analysis for quantitative elemental analysis. X-ray diffraction of the ZnTe:Cu layer was performed on surrogate samples deposited on silicon wafers, to mitigate complications from CdTe and Au.

\section{RESULTS AND DISCUSSION}

During processing the $\mathrm{ZnTe}$ and $\mathrm{Cu}$ are co-evaporated with the intention of distributing $\mathrm{Cu}$ uniformly throughout the layer. Figure 2 displays a high-angle annular dark-field (HAADF) image of the back contact region, and Figure 3 displays elemental contour maps obtained from this same region. The gold contact appears bright in the HAADF image, and the $\mathrm{Pt}$ was introduced during sample preparation. This image contains two large CdTe grains, with a well-defined $\sim 150 \mathrm{~nm}$ ZnTe layer

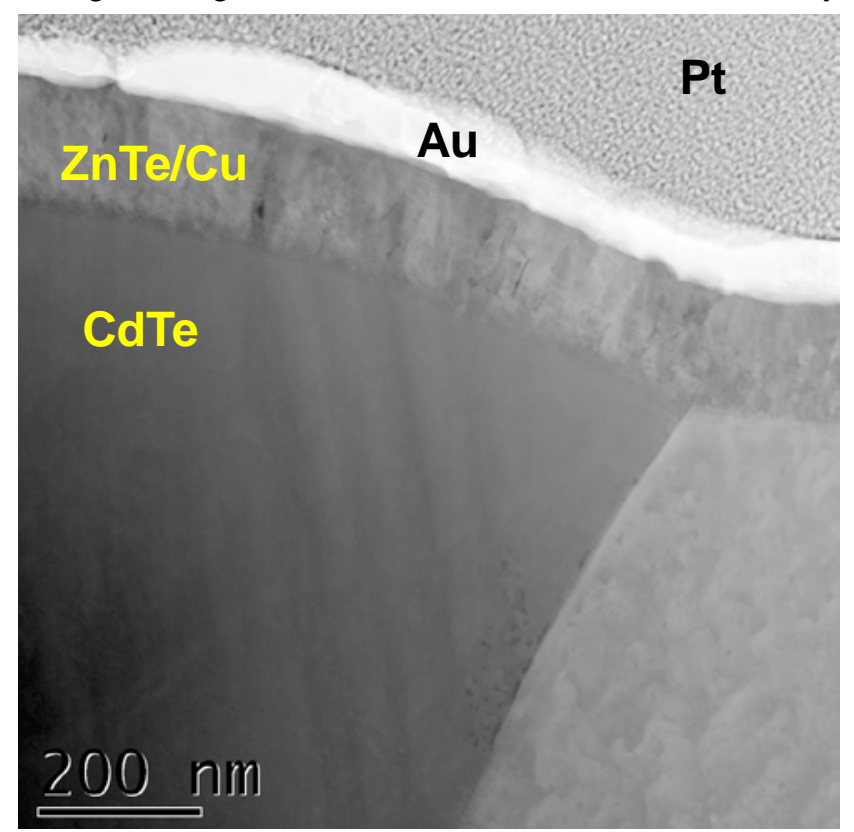

Fig. 2. HAADF image of the back contact region prior to RTP treatment. layer between the absorber and the gold. The individual layers are well defined in the TEM and confirmed by the elemental contour maps in Fig. 3 which have been offset for clarity. As expected, the co-evaporation process distributes the copper uniformly throughout the ZnTe layer. There is no evidence of intermixing with the exception of a small amount of $\mathrm{Cu}$ that appears to penetrate the grain boundary located at the knee in the profile maps.

A critical question is how the composition and structure was altered during the RTP process used for optimal activation. Figure 4 displays a high resolution bright field TEM image of the back contact region after RTP treatment. The ZnTe thickness remains nominally unchanged, but there are substantial changes to its morphology. There appears to be substantial grain growth, in particular one observes twins not unlike those seen in CdTe, particularly in the region adjacent to the gold metallization layer. In contrast the region adjacent to the CdTe layer has a much more amorphous appearance,

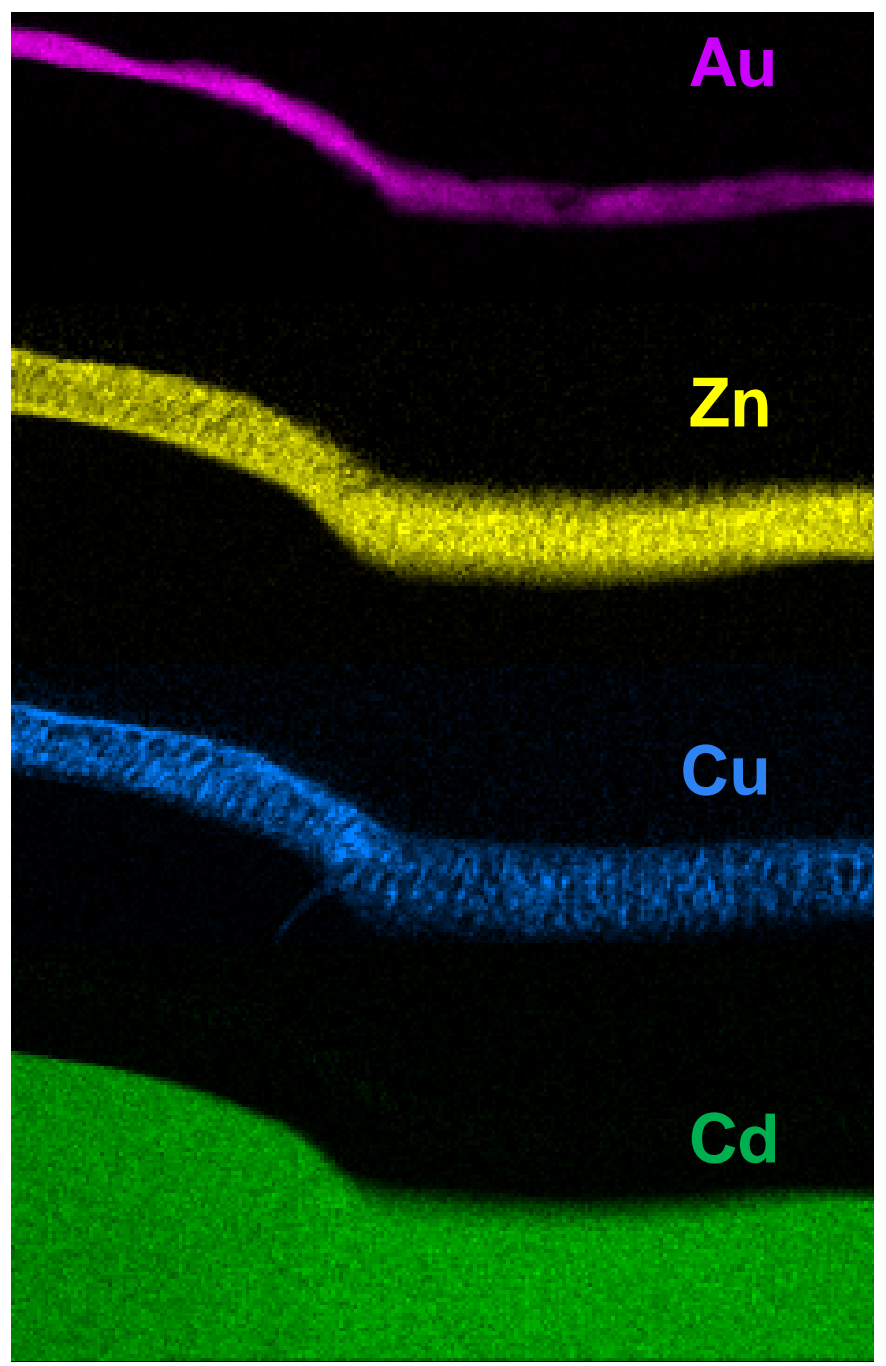

Fig. 3. Elemental contour maps of from the as-deposited back contact region shown in Fig. 2, offset for clarity. 


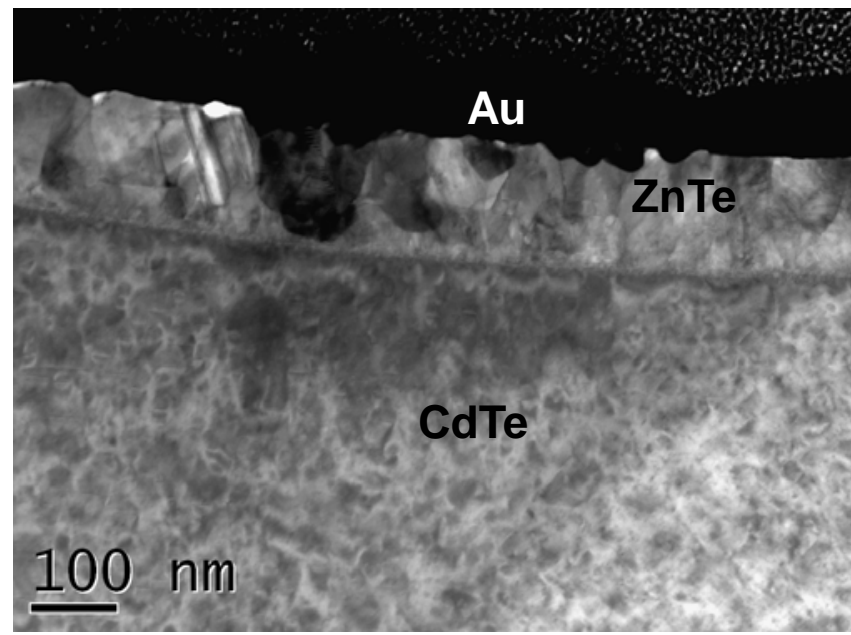

Fig. 4. Bright field HR-TEM after RTP treatment.

and it is suggested that the cause may be Zn-Cd interdiffusion, as previously observed in these contacts by atom probe tomography (APT) [8]. To further assess this the composition was evaluated by performing vertical EDAX line scans through this region, with representative results being shown in Figure 5.

There are several points to take away from the composition profiles shown in Fig. 5. First, the gold layer remains largely intact, with little evidence of interdiffusion or rearrangement. Second, it is observed that there is significant accumulation of $\mathrm{Cu}$ at the $\mathrm{Au} \mid \mathrm{ZnTe}$ interface. This observation is consistent with previous analysis of quantified SIMS results [8], which suggested that up to $80 \%$ of the $\mathrm{Cu}$ deposited in the $\mathrm{ZnTe} \mathrm{Cu}$ layer segregates into the gold during RTP processing. This is perhaps unsurprising as these two metals are completely miscible. Now turning to the ZnTe layer we see two distinct regions that correspond to the morphological distinctions discussed above. It is observed that the composition of first $\sim 100 \mathrm{~nm}$ of this layer adjacent to the gold is approximately stoichiometric ZnTe, corresponding to the region with welldefined crystallites (Fig. 4). In contrast the last $50 \mathrm{~nm}$, the region that appears amorphous in the TEM, show evidence of extensive $\mathrm{Zn}-\mathrm{Cd}$ interdiffusion. In fact the Cd:Zn ratio is approximately 2 in this region. This interdiffusion process appears to occur predominantly within the ZnTe layer, as the composition quickly reverts to stoichiometric CdTe once crossing the ZnTe|CdTe interface.

The changes discussed above are supported by XRD measurements of surrogate films deposited on silicon before and after RTP processing as shown in Figure 6. ZnTe evaporated without $\mathrm{Cu}$ is nanocrystalline as evidenced by its broad lineshape. When co-evaporated with the optimum amount of copper ( 4 wt. \%) the ZnTe signal is sharply attenuated, and the presence of peaks attributed to nanocrystalline copper telluride $\left(\mathrm{Cu}_{\mathrm{x}} \mathrm{Te}\right)$ shows that copper readily reacts even at low temperature. When this sample is then exposed to the optimal RTP treatment, a sharp ZnTe peak reappears, consistent with the grain growth shown in Fig. 4. Note that the $\mathrm{Cu}_{\mathrm{x}} \mathrm{Te}$ peaks remain but are somewhat attenuated.

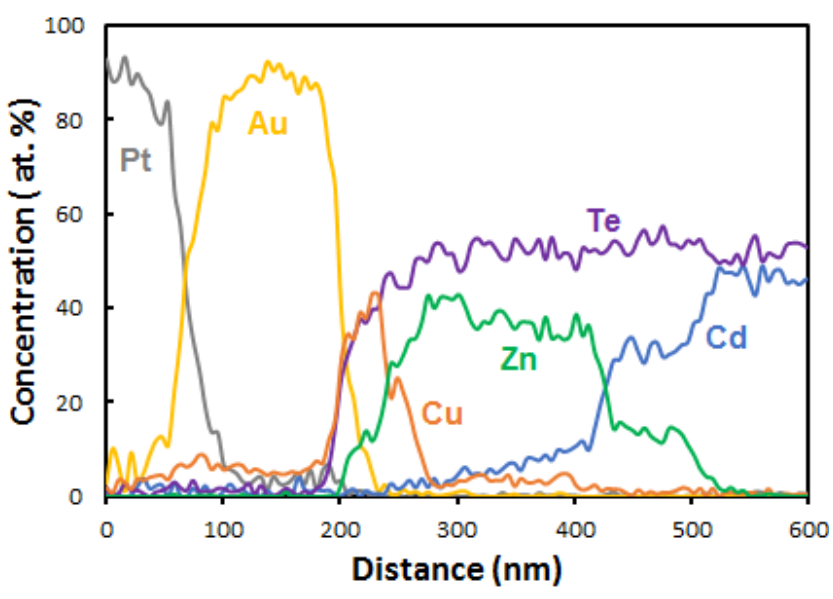

Fig. 5. Representative line scan showing the elemental composition profile through the back contact region.

It is well understood that copper can significantly impact CdTe device performance and reliability, both positively and negatively. As such it is critical to evaluate how the elements are redistributed after RTP. To better understand this question Figures 7 and 8 display HAADF images and associated elemental mapping of the back contact region performed at low and high magnification, respectively. From Figure 7 we see that the Au layer remains largely unaltered, consistent with the line scan shown in Fig. 5. The copper distribution, quite uniform throughout ZnTe as-deposited (Fig. 3), is observed to segregate and aggregate in dramatic fashion after RTP. The majority of the Cu segregates to the Au|ZnTe interface, with much of it present in clusters whose size is on the order of 30-50 nm. Comparing the last two images in Figure 7 one observes that the clusters of $\mathrm{Cu}$ intensity along the Au|ZnTe interface directly correspond to regions of Zn deficiency. While the majority of $\mathrm{Cu}$ migrates to the $\mathrm{Au}$ layer, there are also a few $\mathrm{Cu}$-containing clusters located adjacent to the ZnTe|CdTe interface. These areas are well correlated with regions in the CdTe that are $\mathrm{Cd}$

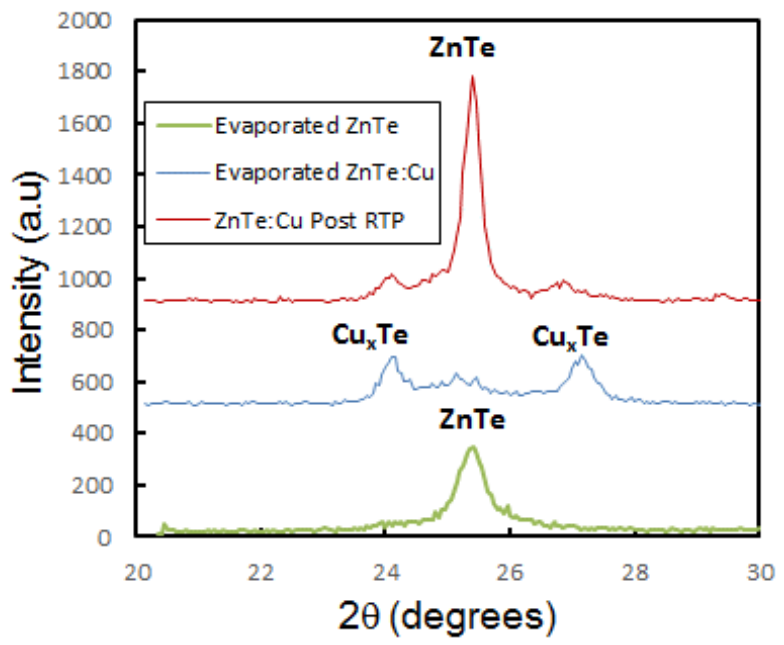

Fig. 6. XRD patterns of evaporated ZnTe, co-evaporated ZnTe:Cu, and after RTP treatment - offset for clarity. 

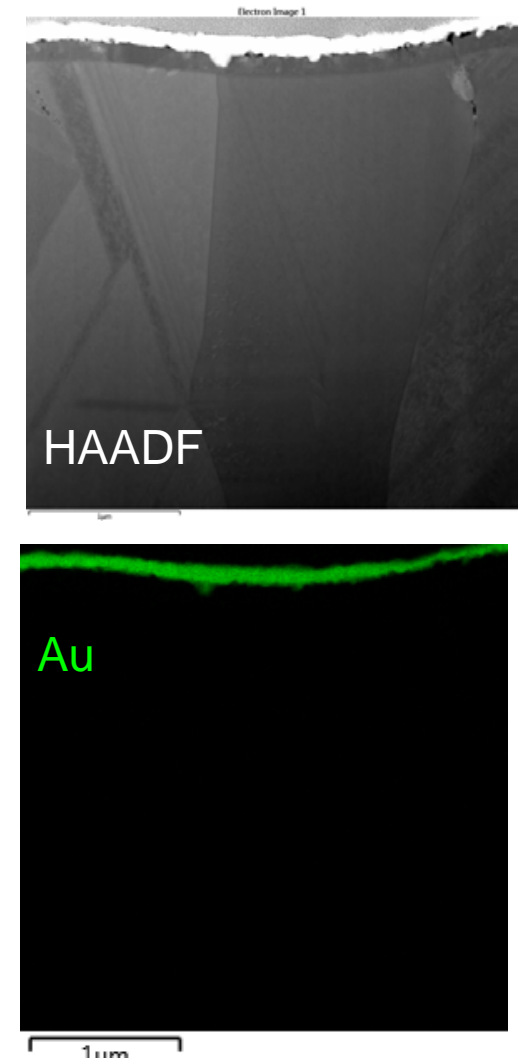

$1 \mu \mathrm{m}$
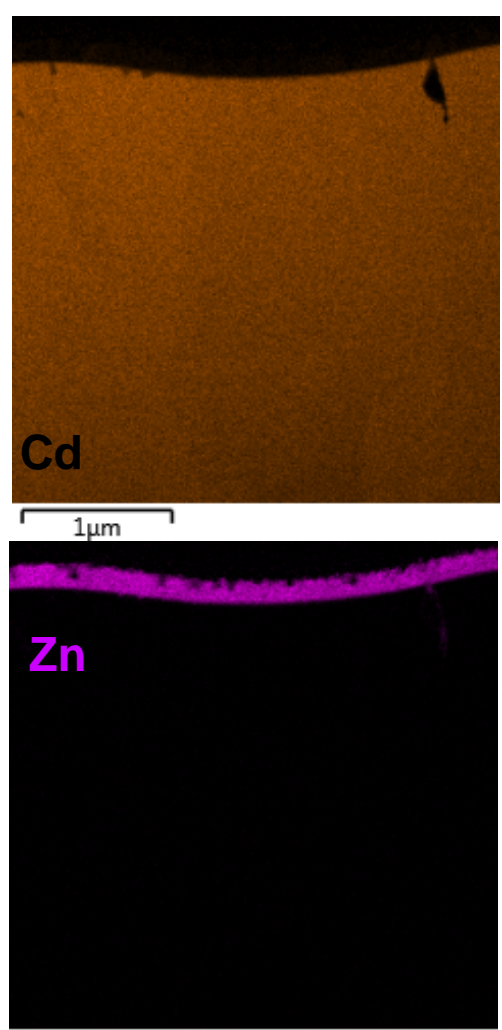

$1 \mu \mathrm{m}$
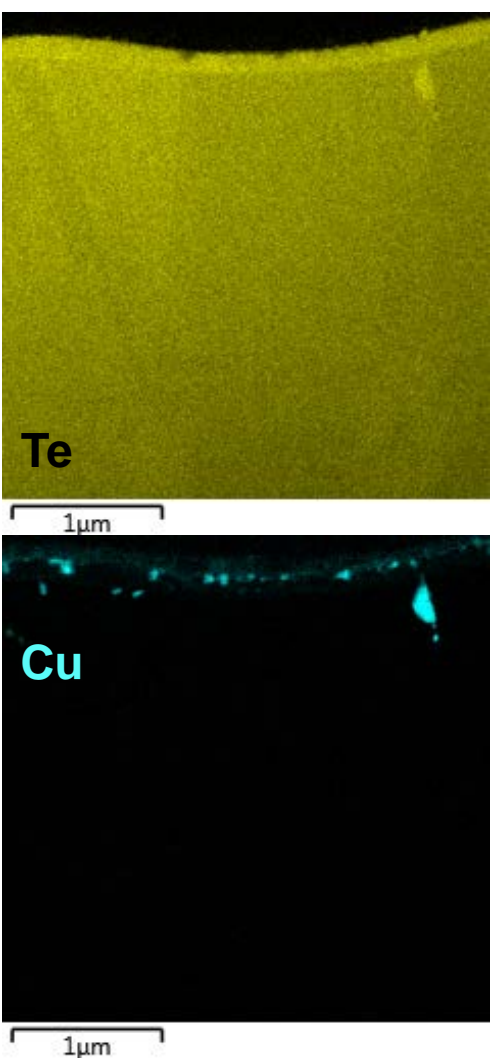

Fig. 7. HAADF image and associated elemental contour maps of the back contact region after RTP treatment.
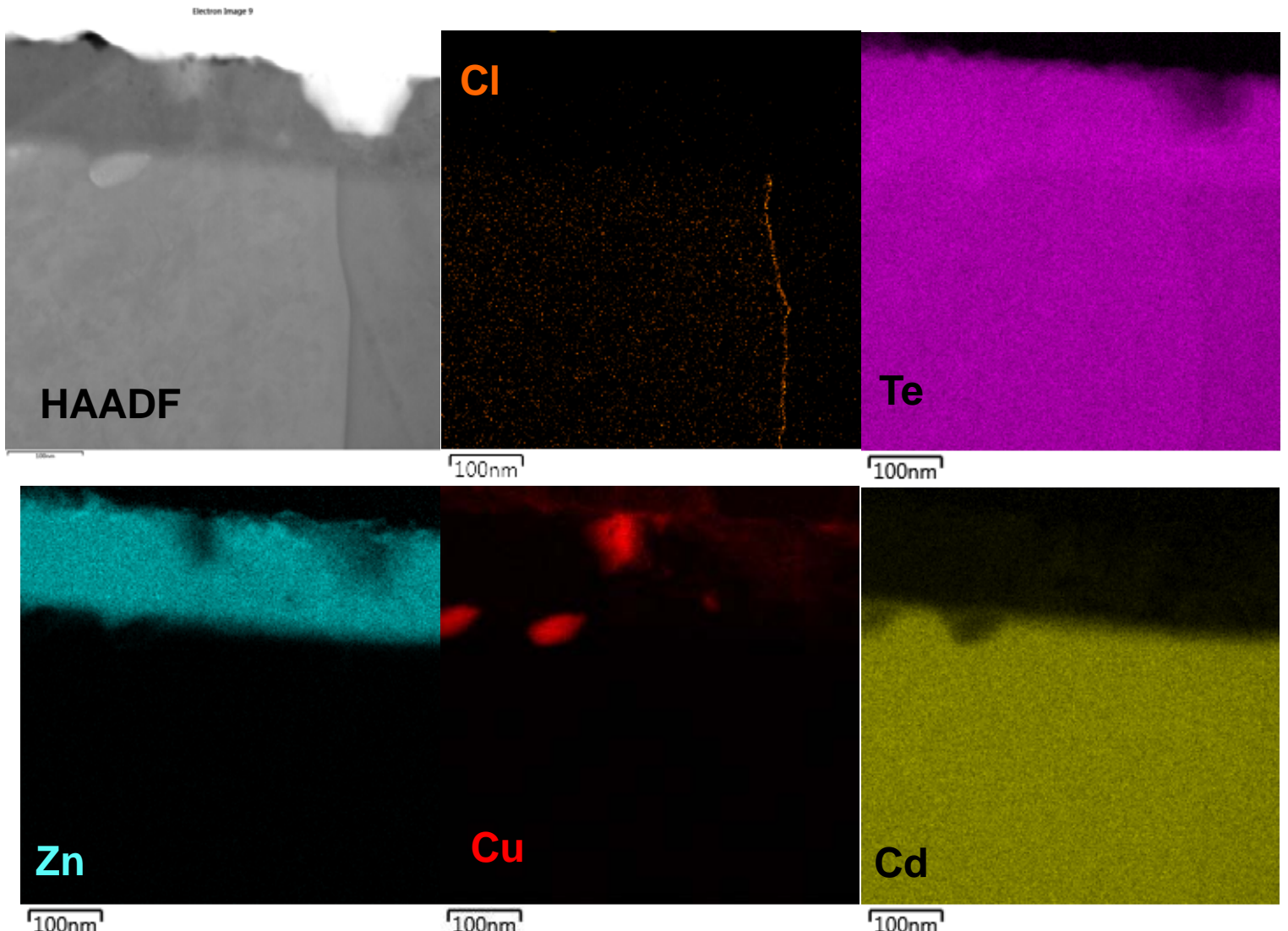

Fig. 8. High resolution HAADF image and associated elemental contour maps of the back contact region after RTP treatment. 
TABLE I

ELEMENTAL COMPOSITION (AT. \%) MEASURED AT INDIVIDUAL POINTS IN FIG. 9

\begin{tabular}{|c|c|c|c|c|c|}
\hline Spot \# & Region & Cd & Te & Zn & Cu \\
\hline 1 & ZnTe layer & 4.2 & 54.2 & 38.5 & 1.7 \\
\hline 2 & CuxTe Cluster & 21.2 & 46.7 & 4.5 & 26.1 \\
\hline 3 & CdTe Grain Boundary & 47.1 & 48.8 & 0.7 & 1.1 \\
\hline 4 & CdTe Grain & 48.7 & 48.9 & 0 & 0.4 \\
\hline
\end{tabular}

deficient, and appear to correspond to the presence of grain boundaries or defects along the CdTe interface.

This correspondence is illustrated more clearly in the high resolution images shown in Fig. 8. There are two regions along the ZnTe|CdTe interface on the left side of the image that are Cd-poor, and this is exactly where Cu-rich aggregates are observed. The $\mathrm{Cl}$ distribution is also shown for this region. Chlorine is detected throughout the CdTe grains, but is particularly concentrated along the vertical grain boundary on the right side of the image as documented previously [11]. The $\mathrm{Cl}$ is introduced during $\mathrm{CdCl}_{2}$ treatment, and it is interesting that little $\mathrm{Cl}$ appears to move into the ZnTe layer during RTP. This suggests that while RTP significantly alters the back contact region, it has minimal impact on the structure and/or composition of the bulk device.

Figure 9 displays a bright field TEM image of the back contact region that contains two of these $\mathrm{Cu}$-rich clusters, which appear as the dark regions located along the grain boundary. Again in this image we observe evidence of a bilayer structure in ZnTe in which there is no clear grain structure in the region adjacent to CdTe, but as one moves away from the CdTe interface well defined grains may be observed. To get a better understanding of the chemical characteristics of the individual regions and interfaces presented in this image the elemental composition was quantified at several spots. Table I summarizes the elemental composition determined at the four spots shown in Fig. 9. Spot 1 is centered within the ZnTe layer, where a significant level of Cd is detected, confirming the Zn$\mathrm{Cd}$ interdiffusion process discussed above. The $\mathrm{Cu}$ content at this position is a fraction of the amount supplied in the asdeposited film, confirming that much of the copper has diffused out of this layer. Spot 2 is centered on the large dark region and elemental analysis confirms that this is indeed a $\mathrm{Cu}_{\mathrm{x}} \mathrm{Te}$ cluster. A significant amount of $\mathrm{Zn}$ is also present, while $\mathrm{Cd}$ is displaced from this region. Spot 3 is centered on the grain boundary (GB) between the two $\mathrm{Cu}_{\mathrm{x}} \mathrm{Te}$ clusters. The two principal impurities are $\mathrm{Cu}$ and $\mathrm{Zn}$. Finally Spot 4 is centered with a CdTe grain, and as expected there are no impurities present. Note that the $0.4 \% \mathrm{Cu}$ detected here is not considered significant, as it is at the detection limits of this technique.

The RTP treatment results in significant improvement in both $\mathrm{V}_{\mathrm{oc}}$ and $\mathrm{FF}[8,9]$. It is postulated that the Cu migration and Zn$\mathrm{Cd}$ interdiffusion observed in this work are responsible for these improvements. It is suggested that RTP creates an efficient tunnel junction for charge transfer, eliminating the
Schottky barrier and improving voltage. Likewise, the passivation of defects by $\mathrm{Cu}_{\mathrm{x}} \mathrm{Te}$ should minimize recombination in the back contact region and facilitate band bending to occur on both sides of the ZnTe|CdTe interface[12]. Analysis of diffusion profiles after RTP found that the effective diffusivity of $\mathrm{Cu}$ during RTP was orders of magnitude lower that what would be expected based on literature [8]. It is suggested that the reason for this is the proclivity of $\mathrm{Cu}$ to react with Te and aggregate in these large clusters may explain these results, as the mobility of large aggregates would be expected to be much less than free atoms.

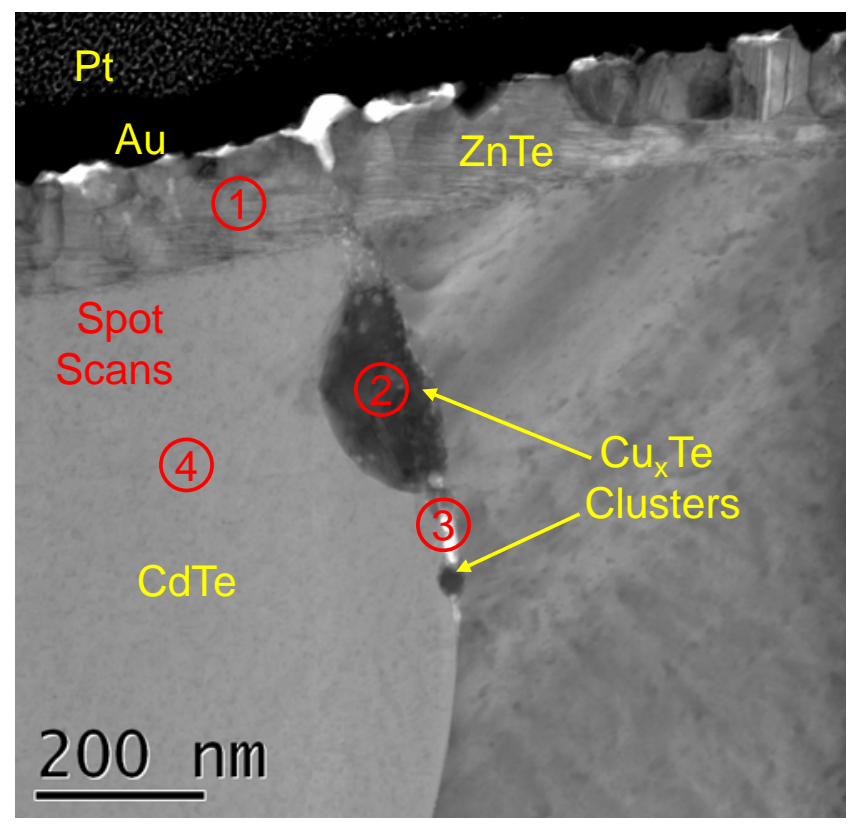

Fig. 9. Bright field TEM image of the Au/ZnTe:Cu/CdTe interface region with numbers and lines indicating the regions where the composition was quantified using EDX point analysis.

\section{CONCLUSIONS}

The structure and composition of the back contact region of high efficiency devices employing a ZnTe:Cu buffer activated by RTP was studied by HRTEM and elemental analysis. Prior to RTP treatment the individual layers are well-defined, with copper distributed uniformly throughout ZnTe. The asdeposited film has a nanocrystalline morphology with both 
ZnTe and $\mathrm{Cu}_{\mathrm{x}}$ Te phases present. After RTP processing a bilayer structure is observed, with well-defined ZnTe crystallites adjacent to the metallization layer, and a more amorphous layer in contact with CdTe in which significant Zn-Cd interdiffusion is observed. Upon RTP treatment copper segregates into aggregates, with the majority of the $\mathrm{Cu}$ moving to the $\mathrm{Au} \mid \mathrm{ZnTe}$ interface. The remaining $\mathrm{Cu}$ forms $\mathrm{Cu}_{\mathrm{x}} \mathrm{Te}$ clusters in $\mathrm{CdTe}$ at defects or grain boundaries near the interface. These changes in structure help explain the physical origins for the dramatic improvements in device performance.

\section{ACKNOWLEDGEMENTS}

We gratefully acknowledge the Bay Area Photovoltaic Consortium for their support of the work performed at CSM under U.S. Department of Energy Award number DEEE0004946. Work at NREL was funded by the U.S. Department of Energy SunShot Foundational Program to Advance Cell Efficiency (F-PACE) under Contract No. DEAC36-08-GO28308. The Loughborough research was funded by UKERC through the EPSRC Supergen SuperSolar Hub.

\section{REFERENCES}

[1] NREL Best Research Cell Efficiency Chart, http://www.nrel.gov/ncpv/images/efficiency_chart.jpg, accessed 5/15/15.

[2] M. Gloeckler, I. Sankin, and Z. Zhao, "CdTe Solar Cells at the Threshold to 20\% Efficiency," IEEE J. Photovolt., vol. 3, pp. 1389-1393, Oct 2013.

[3] A. L. Fahrenbruch, "Exploring back contact technology to increase CdS/CdTe solar cell efficiency," Mater. Res. Soc. Symp. Proc., vol. 1012, pp. 283-290, 2007.

[4] T. A. Gessert, A. R. Mason, P. Sheldon, A. B. Swartzlander, D. Niles, and T. J. Coutts, "Development of $\mathrm{Cu}$-doped ZnTe as a back-contact interface layer for thin- film CdS/CdTe solar cells," J. Vac. Sci. Technol. A, vol. 14, p. 806, 1996.

[5] N. Strevel, L. Trippel, C. Kotarba, and I. Khan, "Improvements in CdTe module reliability and long-term degradation through advances in construction and device innovation," Photovoltaics International, vol. 22, pp. 6674, 2014.

[6] B. E. McCandless and K. D. Dobson, "Processing options for CdTe thin film solar cells," Solar Energy, vol. 77, pp. 839-856, 2004.

[7] S. H. Demtsu, D. S. Albin, J. R. Sites, W. K. Metzger, and A. Duda, "Cu-related recombination in CdS/CdTe solar cells," Thin Solid Films, vol. 516, pp. 2251-2254, 2/29/ 2008.

[8] J. Li, D. R. Diercks, T. R. Ohno, C. W. Warren, M. C. Lonergan, J. D. Beach, et al., "Controlled activation of ZnTe:Cu contacted CdTe solar cells using rapid thermal processing," Solar Energy Materials and Solar Cells, vol. 133, pp. 208-215, 2015.

[9] J. Li, J. D. Beach, and C. A. Wolden, "Rapid thermal processing of ZnTe:Cu contacted CdTe solar cells," in $40^{\text {th }}$ IEEE Photovoltaic Specialists Conference, Denver, CO, 2014, pp. 2360-2365.

[10]D. M. Meysing, C. A. Wolden, M. M. Griffith, H. Mahabaduge, J. Pankow, M. O. Reese, et al., "Properties of reactively sputtered oxygenated cadmium sulfide (CdS:O) and their impact on CdTe solar cell performance," Journal of Vacuum Science \& Technology A, vol. 33, p. 021203, 2015.

[11] A. Abbas, G. D. West, J. W. Bowers, P. M. Kaminski, B. Maniscalco, J. M. Walls, et al., "Cadmium chloride assisted re-crystallization of CdTe: The effect of annealing over-treatment," in Photovoltaic Specialist Conference (PVSC), 2014 IEEE 40th, 2014, pp. 0701-0706.

[12] J. Zhou, X. Wu, A. Duda, G. Teeter, and S. H. Demtsu, "The formation of different phases of $\mathrm{Cu}_{\mathrm{x}} \mathrm{Te}$ and their effects on CdTe/CdS solar cells," Thin Solid Films, vol. 515, pp. 7364-7369, 2007. 